\title{
Aplicaciones de las nanopartículas metálicas en las ciencias veterinarias
}

\author{
Giovany Ortiz-Arana1 $₫ \mathbb{0} ;$ Matín Talavera-Rojas² $\bowtie \mathbb{0} ;$ \\ Valente Velázquez-Ordoñez ${ }^{\otimes}$; jorge Acosta-Dibarrat $2 * \otimes(i)$
}

\begin{abstract}
${ }^{1}$ Universidad Autónoma del Estado de México. Facultad de Medicina Veterinaria y Zootecnia, Programa en Ciencias Agropecuarias y Recursos Naturales. México.

2Universidad Autónoma del Estado de México, Facultad de Medicina Veterinaria y Zootecnia, Centro de Investigación y Estudios Avanzados en Salud Animal, México.

*Correspondencia: jpacostad@uaemex.mx
\end{abstract}

Recibido: Julio 2020; Aceptado: Febrero 2021; Publicado: Junio 2021.

\section{RESUMEN}

Las nanopartículas son materiales que se encuentran a una escala nanométrica menor a $100 \mathrm{~nm}$, se originan de forma natural o por la intervención del hombre y de acuerdo con los elementos que las constituyen adquieren funciones únicas y específicas. En las ciencias veterinarias las nanopartículas metálicas son consideradas una herramienta revolucionaria e innovadora, que permiten entrar a una nueva era en la transformación de los vehículos de medicamentos y vacunas, en el diagnóstico y tratamiento de enfermedades infecciosas y degenerativas, además de mejorar los aspectos zootécnicos de crianza y reproducción de los animales e innovar las herramientas en la vigilancia de la inocuidad de los alimentos de origen animal. En esta revisión se analizaron estudios enfocados en las aplicaciones de las nanopartículas metálicas en las ciencias veterinarias, lo cual brinda un panorama actual de los alcances y limitaciones en el uso de estas herramientas nanotecnológicas en las diferentes áreas del conocimiento veterinario.

Palabras clave: Inocuidad alimentaria; nanobiotecnología; nanomateriales; salud animal (Fuentes: FAO, USDA).

\section{ABSTRACT}

Nanoparticles (NPs) are generally less than $100 \mathrm{~nm}$ in size and originate either naturally or through human activities. According to their constituent elements, NPs exhibit unique and specific functions. In veterinary science, metallic NPs are considered revolutionary and an innovative tool that ushered in a new era in the transformation of drug and vaccine vehicles, diagnosis and treatment of infectious and degenerative diseases, improvement of the zootechnical aspects of animal breeding and reproduction, and innovations in the tools involved in safety monitoring of food products from animal origin. In this review, we focused on studies that highlighted the applications of metallic NPs in veterinary science, thereby providing the current holistic view on the scope and limitations of nanotechnology in different areas of veterinary science.

Keywords: Food safety; nanobiotechnology; nanomaterials; animal health (Sources: FAO, USDA).

Como citar (Vancouver).

Ortiz-Arana G, Talavera-Rojas M, Velázquez-Ordoñez V, Acosta-Dibarrat J. Aplicaciones de las nanopartículas metálicas en las ciencias veterinarias. Rev MVZ Córdoba. 2021; 26(3):e2123. https://doi.org/10.21897/rmvz.2123 


\section{INTRODUCCIÓN}

La nanotecnología es la ciencia que se encarga del estudio de los nanomateriales (1) y la bionanotecnología es la disciplina que se encarga de estudiar los efectos de las interacciones de los nanomateriales con los seres vivos mientras que la bionanomedicina está orientada la utilización de estos nanomateriales en los aspectos biomédicos $(2,3)$.

Las nanopartículas son materiales que se encuentran a una escala nanométrica entre 1 a $100 \mathrm{~nm}$, aunque algunos autores mencionan que pueden medir incluso hasta $1000 \mathrm{~nm}(1,4)$. Cuando los materiales alcanzan una escala nanométrica, estos pueden adquirir propiedades fisicoquímicas únicas en comparación con el material de origen (5), estas propiedades de los nanomateriales van a depender de los elementos que los constituyan (4).

La razón de que las propiedades de los nanomateriales sean tan diferentes a los materiales de origen se debe a dos efectos. El primero está dado por el efecto de superficie, esto se atribuye a que los átomos de los nanomateriales son menos estables, por lo que requieren menor cantidad de energía para unirse en comparación al material de origen. El segundo está dado por el efecto cuántico, debido a que los nanomateriales cuando llegan a una escala nanométrica muestran un comportamiento similar a las propiedades de un átomo individual (6).

Una forma de clasificar a las nanopartículas es de acuerdo con su origen; las nanopartículas de origen natural, que se forman de materiales orgánicos, minerales y las nanopartículas antropogénicas, que se producen por las actividades humanas durante los procesos industriales (7).

Otra manera de clasificar a las nanopartículas es de acuerdo con los materiales que las conforman. En esta categoría de clasificación encontramos distintos tipos; las nanopartículas basadas en carbono constituidas en su mayor porcentaje de este material. Las nanopartículas basadas en metales, que están constituidas por diferentes metales pesados (7), estas a su vez se puede agrupar en cuatro categorías, las nanopartículas metálicas (OD), nanocables y varillas metálicas (1D), láminas y placas metálicas (2D), y nanoestructuras metálicas (3D) (8). Las nanopartículas basadas en dendrímeros, que son formadas por macromoléculas poliméricas sintéticas (peptídicos, lipídicos, polisacarídicos). Finalmente, las nanopartículas compuestas, que son combinaciones entre nanopartículas similares o con materiales de mayor tamaño (7).

Las nanopartículas metálicas tienen diferentes mecanismos de acción sobre las células eucariotas y procariotas, causando daño en la superficie de la pared y membrana celular e inducción de citotoxicidad a través de la generación de especies reactivas de oxígeno (ROS) y la liberación de radicales libres, ocasionando daño en las estructuras intracelulares (mitocondrias, vacuolas, ribosomas) y en biomoléculas (proteínas, lípidos, carbohidratos, ADN). Además, interfieren en la división celular y en la modulación de las vías de transducción de señales para el crecimiento microbiano y la actividad celular. Estos mecanismos de acción pueden ser beneficiosos para el combate de los patógenos, pero también suponen un riesgo para el hospedero $(7,9)$.

Actualmente en las ciencias veterinarias se están evaluando diversos nanomateriales como vehículos de medicamentos y/o vacunas, en el desarrollo de técnicas diagnósticas y tratamientos de diversas enfermedades, en el mejoramiento productivo y reproductivo en los animales e innovaciones en los aspectos de inocuidad lo que otorga un valor agregado a los alimentos de origen animal (10). Entre los nanomateriales más estudiados en las ciencias veterinarias se encuentran las nanopartículas metálicas, tales como las de plata (AgNPs) (11), oro (AuNPs) (12), platino (PtNPs), cobre (CuNPs) (13), selenio (SeNPs) (14), óxido de hierro (Fe2O3NPs) (15), dióxido de titanio (TiO2NPs) (16), óxido de zinc (ZnONPs) (17), entre otras más.

Algunas ventajas de las nanopartículas metálicas es que se pueden sintetizar y modificar con grupos funcionales apropiados que les permiten unirse a medicamentos, anticuerpos, ligandos y sustancias activas (18) por lo que estas características de las nanopartículas les permiten ser consideradas como importantes para las áreas biomédicas (19), aunque también un sinnúmero de ellas presentan riesgos potenciales para la salud y el ambiente. Por lo anteriormente mencionado, el objetivo de esta revisión fue analizar avances científicos y las limitaciones en el uso de las nanopartículas las metálicas en las diferentes áreas de la ciencia veterinaria. 


\section{Aplicaciones en medicina veterinaria}

Una amplia gama de nanopartículas metálicas se está evaluando en medicina veterinaria para el tratamiento de enfermedades infecciosas de origen bacteriano, micótico (15), parasitario (20), viral (21) y no infecciosas como las neoplasias (22). Además, la investigación sobre las nanopartículas metálicas se está centrando en mejorar la respuesta antinflamatoria y el proceso de cicatrización (23), así como, el desarrollo de vacunas, la liberación de fármacos, la innovación de métodos diagnósticos para la detección de biomoléculas (ADN, lípidos, proteínas, metabolitos) y para la identificación de patógenos y adulterantes en los alimentarios (Figura 1) $(24,25)$.

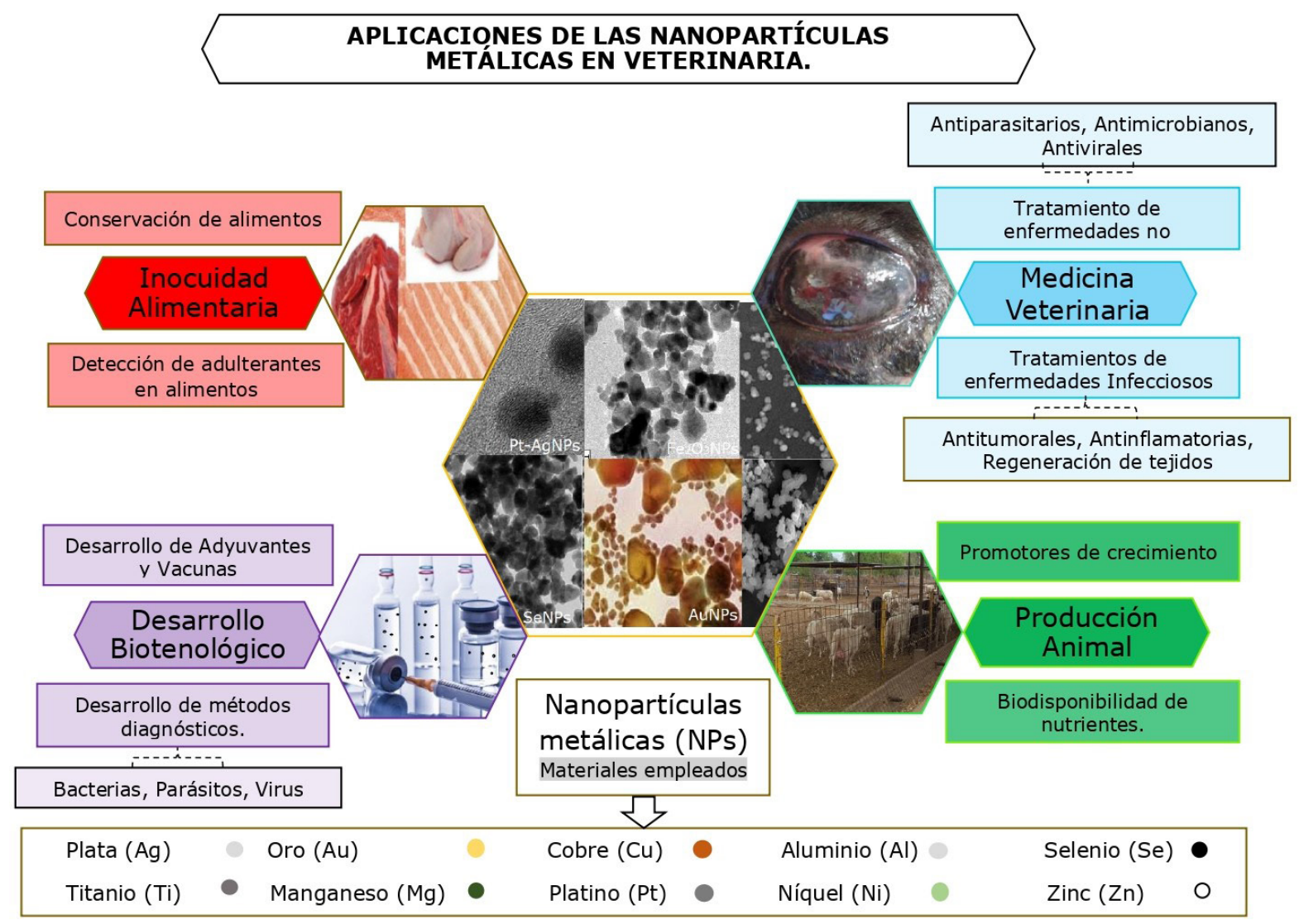

Figura 1. Aplicaciones de las nanopartículas metálicas en las áreas en las ciencias veterinaria.

\section{Propiedades antiparasitarias de las nanopartículas metálicas}

Hoy en día se producen, caracterizan y evalúan diversas nanopartículas metálicas contra diversos endoparásitos y ectoparásitos que son causantes de enfermedades tanto en animales domésticos y acuáticos $(26,27,28,29,30,31,32,33,34)$. Se evaluaron in vitro las AgNPs (con un tamaño de 15-25 nm y de forma esférica) sintetizadas con Azadirachta indica contra larvas y forma adultas de Haemonchus contortus, un parásito muy importante en la producción ovina y caprina, las cuales mostraron inhibición en la eclosión de larvas de $H$. contortus a concentraciones de $1 \mathrm{\mu g} / \mathrm{mL}$ y muerte del parasito adulto a concentraciones de $7.89 \mu \mathrm{g} / \mathrm{mL}$, estos hallazgos evidencian las propiedades antihelmínticas de estas nanopartículas (26).

Con respecto a endoparásitos como el Heterosporis saurida que afecta a animales acuáticos como el pez lagarto (Saurida undosquamis), se están estudiando in vitro los efectos antiparasitarios y citotóxicos de las AuNPs (con un tamaño 11-14 $\mathrm{nm}$ y de forma esférica). Se ha encontrado que estas nanopartículas poseen una actividad esporicida y sin causar un efecto citotóxico en la línea celular (EK-1 de riñón de anguila). Estos resultados muestran que las AuNPs podrían ser consideradas como un agente antimicrosporidial efectivo (27). En otro estudio similar, se evaluaron 
dos tipos de AgNPs obtenidas por diferentes métodos de síntesis (ARGOVIT con tamaño de $35 \mathrm{~nm}$ y UTSA con tamaño de 1-3 nm) contra huevecillos y la fase adulta de Cichlidogyrus spp., los estudios demostraron que ambas AgNPs tuvieron efectos contra este parásito, aunque las AgNPs de UTSA mostraron ser más efectivas con un efecto ovicida y adulticida del $100 \%$ a una concentración de $36 \mu \mathrm{g} / \mathrm{L}$ (28). Los dos estudios anteriores representan las bases de futuras investigaciones en el uso de nanopartículas metálicas para el control de parásitos en peces

Con relación a las ectoparasitosis en peces, los estudios in vitro e in vivo con diversas NPs metálicas (AgNPs, AuNPs y ZnONPs) contra Ichthyophthirius multifiliis, muestran que todas las nanopartículas poseen un efecto protozoacida contra $I$. multifiliis in vitro, aunque las AgNPs y ZnONPs resultaron ser más efectivas. Mientras que los estudios in vivo en trucha arcoíris (Oncoryhnchus mykiss) demostraron que las AgNPs fueron las más eficaces (29).

Actualmente se están buscando nuevas formas para controlar algunos ectoparásitos que son responsables de afectar la productividad de los animales $(20,30)$ y otros que son responsables de transmitir diversas enfermedades tanto a los humanos como a los animales (31). En un estudio contra las garrapatas de la especie Rhipicephalus microplus que afectan al ganado bovino, se evaluaron in vitro las ZnONPs (con un tamaño de 20-65 nm con formas esféricas y hexagonales) sintetizadas con Lobelia leschenaultiana, estas nanopartículas mostraron un efecto garrapaticida de $100 \%$ a concentraciones de $0.008 \mathrm{mg} /$ $\mathrm{ml}$ (20). También se probaron las AgNPs (con un tamaño de 25-60 nm y de forma esférica) sintetizada con Mimosa púdica contra larvas de garrapata de $R$. microplus, determinando que las AgNPs tienen un efecto garrapaticida a concentraciones de $8.98 \mathrm{mg} / \mathrm{L}$ (32).

En una nueva medida para controlar las diferentes etapas del ciclo biológico de algunos mosquitos del orden Díptera como Aedes aegypti, Anopheles stephensi y Culex quinquefasciatus son vectores de importantes enfermedades de humanos y animales, se están empleando las nanopartículas $\mathrm{Au}, \mathrm{Ag}, \mathrm{Cu}, \mathrm{Ni}, \mathrm{ZnO}, \mathrm{TiO}_{2}$ a diferentes concentraciones, los resultados de las investigaciones demuestran que todas las nanopartículas poseen un efecto biocida (ovicidas, pulpicidas, larvicidas y adulticidas) en cada especie de mosquitos (31). Para prevenir las miasis en el ganado bovino, se está evaluando in vitro I las ZnONPs sintetizadas con Lobelia leschnaultiana contra larvas de la especie Lucilia sericata, estas nanopartículas presentaron un efecto larvicida a concentraciones de $0.78 \mathrm{mg} / \mathrm{L}$ (33). También se ha ensayado la acción las nanopartículas $\mathrm{TiO}_{2} \mathrm{NPs}$ (con un tamaño de 25$110 \mathrm{~nm}$ y formas irregulares) sintetizadas con Catharanthus roseus contra la mosca hematófaga Hippobosca maculata demostrando un efecto larvicida y adulticida a una concentración de $7.09 \mathrm{mg} / \mathrm{L}(16)$.

Para el tratamiento de la pediculosis en humanos $y$ ovinos se realizaron ensayos in vitro con AgNPs (con un tamaño de $59.52 \mathrm{~nm}$ y de forma esférica) sintetizadas con Lawsonia inermis contra Pediculus humanus capitis y Bovicola ovis, donde estas nanopartículas expresaron un efecto biocida a una concentración de $1.33 \mathrm{mg} / \mathrm{L}$ y $1.41 \mathrm{mg} / \mathrm{L}$ respectivamente (34). Mientras que las $\mathrm{TiO}_{2}$ NPs mostraron el mismo efecto contra B. ovis a una concentración de $6.56 \mathrm{mg} / \mathrm{L}(16)$.

Aunque todos los estudios anteriores proponen a las NPs metálicas como una alternativa en el control de parásitos en animales acuáticos y terrestres, los autores coinciden que existen riesgos de citotoxicidad y genotoxicidad debido a la inducción de apoptosis y necrosis tisular ocasionadas por la generación de ROS inducidas por las NPs. El daño celular generado por las NPs va a depender de diversos factores como; la eficacia de los mecanismos antioxidantes, la eficiencia de los sistemas de reparación de ADN, la propensión apoptótica y la capacidad de resistencia celular y por las características propias de las nanopartículas como el tamaño, forma, carga superficial, revestimiento superficial, solubilidad, concentración, el modo entrada y la estabilidad de las partículas $(16,21,26,27,28,29,30,31,32,33,34)$.

\section{Propiedades antimicrobianas yantimicóticas de las nanopartículas metálicas}

Entre las alternativas para evitar el uso indiscriminado de antimicrobianos, se encuentran las nanopartículas de $\mathrm{Fe}_{2} \mathrm{O}_{3} \mathrm{NPs}$ (con un tamaño de $80 \mathrm{~nm}$ ) obtenidas por síntesis biológica con Candida albicans que presentan efecto antimicrobiano contra Thicohyton verrecosum, $T$. mentagrophyte y Dermatophilus congolensis que causan afecciones dermatológicas en el ganado bovino (15), también las ZnONPs (con un tamaño $60 \mathrm{~nm}$ y forma hexagonal), han demostrado efecto antimicrobiano contra $T$. mentagrophyte, Microsporum canis, C. albicans y Aspergillus fumigatus (35). 
Las nanoparticulas de $\mathrm{Au}, \mathrm{Ag}, \mathrm{Cu}$ y $\mathrm{Pt}$ se han probado in vitro contra los microorganismos implicados en la mastitis bovina, los resultados demuestran que las AgNPs y CuNPs poseen efecto contra $S$. aureus, E. coli, S. uberis, C. albicans y C, krusei, (13). En un estudio similar enfocado en agentes causales de mastitis en caprinos, determinaron que las AgNPs (de tamaño 1-21 nm) poseen actividad antimicrobiana contra cepas multirresistentes de $S$. aureus y $P$. aeruginosa atribuyendo dicho efecto a la generación de especies reactivas de oxígeno (ROS), malondialdehído (MDA) y a la pérdida de proteínas y azúcares estructurales que componen el citoplasma y la membrana celular (36).

En el tratamiento de la endometritis y metritris en vacas lecheras asociadas a cepas multirresistentes de Prevotella melaninogenica y Arcanobacterium pyogenes las AgNPs (con un tamaño de 10 $\mathrm{nm}$ de forma esférica) demostraron inhibición en el desarrollo bacteriano y en la formación de biofilms por lo cual podrían constituir una alternativa en los tratamientos de enfermedades reproductivas asociados con dichos patógenos en los hatos lecheros (37). Las AuNPs (con un tamaño de $25 \mathrm{~nm}$ de forma esférica) combinadas con láser in vitro tienen efecto inhibitorio contra Corynebacterium pseudotuberculosis agente causal de la Linfadenitis caseosa en ovinos y caprinos (38). Mientras que las nanopartículas AgNPs (con un tamaño $\leq 100$ $\mathrm{nm}$ ) inhibieron el desarrollo de Moraxella ovis multirresistentes obtenidos de casos clínicos de Queratoconjuntivitis ovina a concentración de $10.87 \mu \mathrm{g} / \mathrm{L}$ (39). Las AgNPs (con un tamaño de 15-35 nm) sintetizadas con Azadirachta indica y funcionalizadas con aceites vegetales tienen efecto bactericida contra $E$. coli y $S$. aureus y fungicida contra Aspergillus fumigatus y $A$. niger , todos patógenos de interés clínico en humanos y animales (40). También se evaluó la actividad antimicrobiana de las AgNPs y nanocables de plata (AgNWs) sintetizadas con Camellia sinensis, contra $E$. coli y $S$. aureus, resultando con mayor capacidad inhibitoria de los AgNWs contra $S$. aureus, y un efecto similar de ambas nanopartículas contra $E$. coli con concentraciones de $25.8 \mathrm{mg} / \mathrm{mL}$ (41).

Con respecto a patógenos bacterianos que afectan a peces como Streptococcus iniae, Lactococcus garvieae, Yersinia ruckeri, Aeromonas hydrophila en todos estos casos las AgNPs (con un tamaño de
$18 \mathrm{~nm}$ ) inhiben su crecimiento (42). En un estudio similar evaluaron nanopartículas comerciales de C-ZnONPs y C-AgNPs (de tamaño $100 \mathrm{~nm}$ ) y AgNPs (de tamaño 11-39 nm con formas esféricas) contra $A$. hydrophila, $A$. salmonicida subsp. salmonicida, Edwardsiella ictaluri, E. tarda, Francisella noatunensis subsp. orientalis, Yersinia ruckeri y Aphanomyces invadans, demostrando que todas las nanopartículas empleadas tienen una actividad antimicrobiana, aunque las ZnONPs fue la única capaz de inhibir a Y. ruckeri, En este estudio las AgNP fueron las que mostraron menores efectos citotóxicos en las líneas celulares utilizadas (43).

\section{Propiedades antivirales de las nanopartículas metálicas}

Las AgNPs (de un tamaño $\leq 100 \mathrm{~nm}$ ) estabilizadas con polivinilpirrolidona (PVP) diminuye las secuelas en perros con Distemper canino con o sin signos neurológicos además de no presentar signos de toxicidad al tratamiento (21). En otro estudio se evaluó in vitro la acción de las nanopartículas MgONPs (con un tamaño de $\leq 50$ $\mathrm{nm}$ ) contra el virus de la fiebre aftosa (FMDV), estas nanopartículas presentaron un efecto de inhibitorio en la etapa de adhesión y penetración celular del virus (44).

\section{Propiedades antitumorales de las nanopartículas metálicas}

Desde el punto de vista epidemiológico, las enfermedades neoplásicas van en aumento, ocasionando un incremento en la mortalidad en los animales domésticos. Por tal razón se realizan estudios in vitro e in vivo para evaluar la acción de las AuNPs con glutatión (Au-GHS) y en combinación con doxorrubicina Au-GHS-Dox contra fibrosarcoma felino, dichas nanopartículas tuvieron efecto apoptótico sobre tres (FFS1WAW, FFS1 y FFS3) de las cuatro las líneas de celulares tumorales evaluadas $(23,45)$. Mientras en el estudio in vivo en felinos se observó reducción del tamaño tumoral con mínima bioacumulación en hígado, bazo, riñón y corazón sin afectar las funciones normales ni provocar los aumentos sanguíneos de BUN, AST y ALT (46).

Las nanopartículas magnéticas de óxido de hierro $\mathrm{Fe}_{3} \mathrm{O}_{4}$ han sido utilizadas por inoculación directa en el tratamiento del adenocarcinoma de glándula mamaria en felinos produciendo reducción en el tamaño de la masa tumoral. Los estudios 
citológicos revelaron que las células tumorales endocitaban masivamente a las nanopartículas magnéticas produciendo la muerte celular (47). También se observó que las nanopartículas de óxido de grafeno reducido y plata (rGO-Ag) sintetizadas con la planta Tilia amurensis lisan las células de las líneas tumorales de cáncer de ovario (A2780) (48).

\section{Propiedades antinflamatoria y proceso cicatrización}

Loa efectos de regeneración y cicatrización de las AgNPs sintetizadas con $A$. indica y funcionalizadas con aceites vegetales se han evaluado en lesiones en orejas de conejos, observando cicatrización más rápida que con los tratamientos convencionales de antibióticos y antinflamatorios (40). La aplicación directa de una solución de $90 \mu \mathrm{M}$ de AgNPs (con un tamaño de $14.5 \pm 1.2 \mathrm{~nm}$ ) en la cavidad peritoneal antes de un cierre quirúrgico del abdomen en ratones, reduce las adherencias peritoneales y controla el proceso inflamatorio, observando por inmunohistoquímica una disminución expresión del IFN-Y en las muestras de tejido peritoneal. Además estas nanopartículas provocaron disminución de la expresión del TNF-a una importante citocina proinflamatoria en las líneas celulares de macrófagos de ratón RAW264.7 y J774.1 (23).

\section{Aspectos zootécnicos e inocuidad alimentaria}

Las aplicaciones de las nanopartículas metálicas en la producción animal e inocuidad alimentaria se enfocan en mejorar los aspectos de la nutrición animal (desde la ingestión de los alimentos hasta la captación y aprovechamiento de los nutrientes), así como los aspectos reproductivos, la trazabilidad de los productos de origen animal y bioseguridad de los alimentos (49). Para la detección de diversos compuestos que alteran la inocuidad de los alimentos como; aflatoxinas, micotoxinas, bacterias que originan enfermedades transmitidas por alimentos (ETDA) (Salmonella, E. coli 0157:H7, Campylobacter jenuni), toxinas bacterianas como la toxina colérica, adulterantes químicos (melamina, oxalatos, ácido benzoico) y residuos de antibióticos (cloranfenicol y penicilinas) (50).

Se ha empleado a las AgNPs como aditivo antimicrobiano en el agua de bebida para promover el crecimiento en aves de engorda, sin evidenciar ningún efecto sobre el crecimiento, ni cambios metabólicos en las aves, ni en la composición de la microbiota intestinal (51). Por otro lado, la inoculación de AgNPs solas y en combinación con aminoácidos esenciales como la treonina y no esenciales como la cisteína en el saco aéreo del huevo durante el desarrollo embrionario de pollos mejora su estado inmunológico sin alterar su desarrollo (52).

En el tratamiento de la deficiencia de selenio en los pequeños rumiantes se han probado las nanopartículas de selenito de sodio SeNPs, para mejorar la disponibilidad de absorción de este mineral a nivel del abomaso y duodeno (53), además se evaluaron las interacciones de las SeNPs con la homeóstasis de hierro mediante la expresión de transferrina y las proteínas de unión de la transferrina permitiendo la captación y concentración de hierro en plasma (14).

Actualmente se buscan nuevas alternativas para mejorar o sustituir algunos conservadores en alimentos, por tal motivo, se realizaron estudios comparativos entre las propiedades antibacterianas del óxido de zinc ( $\mathrm{ZnO}$ ) y las nanoparticulas de $\mathrm{ZnO}$ (ZnONPs), demostrando que las ZnONPs tienen efecto contra Salmonella typhimurium, S. aureus y E.coli, lo cual sugiere que estas nanopartículas podrían ser empleadas como agentes conservantes, aunque se necesitan estudios sobre los riesgos nanotoxicológicos de estos nanomateriales (54). En un estudio similar se determinó el efecto antifúngico que tienen las nanopartículas de $\mathrm{Fe}_{2} \mathrm{O}_{3}$ (tamaño $45 \mathrm{~nm}$ ) y $\mathrm{Fe}_{3} \mathrm{O}_{4}$ (tamaño $9 \mathrm{~nm}$ ) contra Aspergillus flavus aislado de alimentos destinados para la engorda de pollos, determinado que las nanopartículas de $\mathrm{Fe}_{2} \mathrm{O}_{3}$ tienen un efecto inhibitorio mayor que las de $\mathrm{Fe}_{3} \mathrm{O}_{4}$ (55).

Las propiedades electrocatalíticas de las nanopartículas de oro (AuNPs) fueron utilizadas para el desarrollo de un sistema de detección de E. coli 0157: $\mathrm{H7}$ en muestras de carne molida y agua de grifo. El sistema emplea perlas magnéticas conjugadas con un anticuerpo contra $E$. coli 0157:H7 (MBs-pECAb) y doble marcación con un anticuerpo secundario (AuNPs-sECAb) utilizando las propiedades electrocatalíticas de las AuNPs para realizar la detección y cuantificación por un sistema de cronoamperometría. Este sistema detecta bajas concentraciones bacterianas de $10^{2}-10^{5} \mathrm{UFC} / \mathrm{mL}$ en el $91.3 \%$ y $94.8 \%$ de las muestras de carne y de agua grifo respectivamente, lo que aunado la rapidez y sencillez de la técnica, muestra su potencialidad para ser utilizada en otros alimentos y fuentes de agua (56). 
En el desarrollo de nuevos métodos para la detección de compuestos adulterantes en los alimentos se realizan estudios con sondas de AuNPs (con un tamaño de 10, 40 y $80 \mathrm{~nm}$ ) estabilizadas con citrato trisódico para la detección de melanina en leche cruda (57). También para la detección y recuperación de micotoxinas en los alimentos, se emplean nanopartículas magnéticas de $\mathrm{Fe}_{2} \mathrm{O}_{3} \mathrm{NPs}$ (con un tamaño de 100 y $200 \mathrm{~nm}$ ) funcionalizadas con grupos aminos y anticuerpos monoclonales para la detección de aflatoxina B1 (AFB1) y zearalenona (ZEN), este nuevo sistema permite además recuperar cerca de $90-92 \%$ de las AFB1 y $81-88 \%$ de la ZEN en los productos de maíz y en otros alimentos (58).

\section{Desarrollo de adyuvantes y vacunas}

En el desarrollo de nuevas de vacunas y adyuvantes para estimular la respuesta inmune celular contra microorganismo intracelulares y células tumorales, se han probado las nanopartículas de oro soportado con ultraesferas de óxido de grafeno y blindadas con ovoalbumina (UsGO-Au@OVA), estas nanopartículas han demostrado estimular la respuesta inmune celular a través de la secreción a TNF-a e IFN- $\gamma$ en las líneas celulares macrófagos de ratón RAW264.7 (59). Mientras que en otro estudio evaluaron de manera in vitro e in vitro las AgNPs sintetizadas con Eucaliptus como adyuvante en la vacuna contra la rabia, estas nanopartículas mostraron resultados favorables al presentar mínimos efectos adversos en las líneas celulares L929 y en los modelos murino y canino con respecto al adyuvante de aluminio que contiene la vacuna convencional (60). La conjugación del antígeno viral proveniente del agente causal de la gastroenteritis transmisible porcina (TGS) con las SeNPs y AuNPs produce un inmunógeno que genera buena respuesta inmune humoral y celular en conejillos de india, aumentando las concentraciones plasmáticas de INF- $\gamma, \mathrm{IL}-1 \beta, \mathrm{IL}-6$, lo que conduce a la activación de los macrófagos y células linfoides lo que promueve el aumento de la expresión del péptido viral antigénico en la superficie de las células presentadoras de antígeno contribuyendo así a su presentación efectiva del hacia a los linfocitos T CD4 y CD8 generando una fuerte respuesta humoral y celular (61).

\section{Diagnóstico de enfermedades virales y bacterianas}

En el desarrollo de una nueva técnica de diagnóstico para la Fiebre Aftosa, las AuNPs han sido empleadas como biosensores capaces de identificar específicamente a los tres serotipos del virus (O, A y SAT2). Esto puede ser considerado como una herramienta en el diagnóstico de esta enfermedad en zonas endémicas (12). Mientras para la detección del virus de la Fiebre Porcina Clásica (CSFV), se han diseñado sondas de nanoflare utilizando AuNPs conjugadas con secuencias específicas del virus, que puede reconocer y detectar concentraciones bajas del virus (50 pg $/ \mu \mathrm{L})$ a partir de tejidos (62).

Un nuevo método de diagnóstico para la detección de partículas virales del síndrome respiratorio y reproductivo porcino (PRRSV), el cual emplea biosensores ópticos y nanofotónicos constituidos por dos arquitecturas de biomoléculas; la primera arquitectura es un anticuerpo específico PRRSV marcado con un fluoróforo y unida a una proteína marcada con un punto cuántico y la otra arquitectura marcada con AuNPs, estos biosensores permitieron detectar hasta tres partículas del virus suspendidas en una muestra (63).

Se han desarrollado técnicas rápidas de diagnóstico, sensibles y específicas para enfermedades de origen bacteriano como el nano PCR que utiliza sondas de AuNPs con el fin de detectar la región del gen IS711 de Brucella spp., siendo capaz de identificar concentraciones de DNA bacteriano de 1.09 pg/L (64). Para el diagnóstico de algunas enfermedades que afectan a los peces, se han desarrollado inmunoensayos específicos con AuNPs recubiertas con anticuerpos policlonales para la detección de Aeromona salmonicida, lo que ha permitido detectar a esta bacteria a concentraciones de $1 \times 10^{4} \mathrm{UFC} / \mathrm{mL}$ a partir de bazo o riñón de peces que presentaban signos clínicos de forunculosis (65).

En conclusión, los recientes avances científicos y el desarrollo de nuevas tecnologías han permitido la implementación de la nanotecnología en las diferentes ciencias incluyendo la veterinaria. Actualmente existen cientos de publicaciones referentes a la síntesis y evaluación de diversas nanopartículas metálicas con la finalidad de determinar sus propiedades únicas y aprovecharlas en las diferentes disciplinas. Dichas propiedades de las nanopartículas les permitieron ser consideradas en la ciencia veterinaria como candidatas a formar parte de inmunógenos y medicamentos con la finalidad de establecer estrategias para la prevención $y$ control de enfermedades infecciosas y degenerativas a corto, mediano y largo plazo. 
A pesar de las ventajas que pueden suponer el uso de nanopartículas metálicas, algunos estudios in vitro e in vivo evidencian que estas presentan un efecto de bioacumulación en las células y tejidos animales, lo que puede ocasionar daño en las células eucariotas derivado de sus mecanismos de acción, sin embargo, para alcanzar un efecto de citotoxicidad en un organismos vivo, se necesitan concentraciones altas de las nanopartículas metálicas en un tiempo prolongado en comparación a las concentraciones de los materiales de origen. Otras aplicaciones de las nanopartículas metálicas están el desarrollo de métodos diagnósticos para la detección de diversas patologías y al mismo tiempo ser ocupadas como herramientas complementarias para la vigilancia de la inocuidad de los alimentos. El desarrollo de estas herramientas diagnósticas permitirá una mayor rapidez en la generación de resultados, pruebas más sensibles y específicas y de fácil acceso para los profesionistas a nivel de campo. Las aplicaciones de la nanotecnología en las ciencias veterinarias aún están en sus fases iniciales y requieren del desarrollo de más investigación para estimar los posibles efectos adversos y comprender sus mecanismos de acción, con el fin de pronosticar los riesgos adicionales para los seres humanos, animales y el medio ambiente.

\section{Conflicto de interés}

Los autores declaramos que no existen conflictos de interés.

\section{Agradecimientos}

Al CONACYT-México por la beca (2019-00003702NACF-09642) otorgada al primer autor para la realización de sus estudios de Doctorado. Al financiamiento otorgado a la Red "Nanotecnología y Salud" SEP 2015.

\section{REFERENCIAS}

1. Buzea C, Pacheco II, Robbie K. Nanomaterials and nanoparticles: Sources and toxicity. Biointerphases 2007; 2(4):MR17-MR71. https://doi.org/10.1116/1.2815690

2. Appasani K. BioNanoMedicine: A nanotechnology platform for the 21st century. Expert Rev Mol Diagn 2005; 5(6):839-840. https://doi.org/10.1586/14737159.5.6.839

3. Vazquez-Muñoz R, Huerta-Saquero A. Nanomateriales con actividad microbicida: una alternativa al uso de antibióticos. Mundo Nano 2014 ; 7(13):37-47. https://doi.org/10.22201/ ceiich.24485691e.2014.13.48707

4. Mohanraj VJ, Chen Y. Nanoparticles - A Review. Trop J Pharm Res 2006; 5(1):561-573. https://doi.org/10.4314/tjpr.v5i1.14634

5. Kim JS, Kuk E, Yu KN, Kim JH, Park SJ, Lee $\mathrm{HJ}$, et al. Antimicrobial effects of silver nanoparticles. Nanomedicine 2007; 3(1): 95-101. https://doi.org/10.1016/j. nano.2006.12.001
6. Roduner E. Size matters : why nanomaterials are different. Chem Soc Rev 2006; 35(7)583592. https://doi.org/10.1039/B502142C

7. Frejo M, Díaz M, Lobo M, García J, Capó M. Nanotoxicología ambiental: retos actuales. Med Balear 2011; 26(2):36-46. http://ibdigital.uib. es/greenstone/collect/medicinaBalear/index/ assoc/Medicina/ Balear/2011v26n/2p036. dir/Medicina Balear 2011v26n2p036.pdf

8. Zhang XF, Liu ZG., Shen W, Gurunathan S. Silver Nanoparticles: Synthesis, characterization, properties, applications, and therapeutic approaches. Int J Mol Sci 2016; 17(9):1534. https://doi.org/10.3390/ijms17091534

9. Dakal TC, Kumar A, Majumar, RS, Yadav V. Mechanism basis of antimicrobial action of silver nanoparticles. Front Microbiol 2016; 7:1831. https://doi.org/10.3389/ fchem.2020.00341

10. Bai DP, Lin $X Y$, Huang $Y F$, Zhang XF. Theranostics aspects of various nanoparticles in Veterinary Medicine. Int J Mol Sci 2018; 19(11):3299. https://doi.org/10.3390/ jjms19113299 
11. Elemike EE, Onwudiwe, Ekennia AC, Sonde $\mathrm{CU}$, Ehiri RC. Green synthesis of $\mathrm{Ag} / \mathrm{Ag}_{2} \mathrm{O}$ nanoparticles using aqueous leaf extract of Eupatorium odoratum and its antimicrobial and mosquito larvicidal activies. Molecules 2017; 22(5):674. https://doi.org/10.3390/ molecules 22050674

12. Hamdy ME, Del Carlo M, Hussein HA, Salah TA, El-Deeb AH, et al. "Development of gold nanoparticles biosensor for ultrasensitive diagnosis of foot and mouth disease virus. J Nanobiotechnology 2018; 16(1):48. https:// doi.org/10.1186/s12951-018-0374-x

13. Wernicki $A$, Puchalski $A$, Urban-Chmiel $R$, Dec $M$, Stęgierska $D$, Dudzic $A$, et al. Antimicrobial properties of gold, silver, copper and platinum nanoparticles against selected microorganisms isolated from cases of mastitis in cattle. Med Weter 2014; 70(9):564-567. http://www. medycynawet.edu.pl/images/stories/pdf/ pdf2014/092014/201409564567.pdf

14. Kojouri GA, Jahanabadi S, Shakibaie M, Ahadi AM, Shahverdi AR. Effect of selenium supplementation with sodium selenite and selenium nanoparticles on iron homeostasis and transferrin gene expression in sheep: A preliminary study. Res Vet Sci 2012; 93(1):275-278. https://doi.org/10.1016/j. rvsc.2011.07.029

15. Hassan AA, Oraby $\mathrm{NH}$, El-Dahshan EME, Ali M. Antimicrobial potential of iron oxide nanoparticles in control of some causes of microbial skin affection in cattle. Eur J Acad Essays 2015; 2(6):20-31. https://www. semanticscholar.org/paper/AntimicrobialPotential-of-Iron-Oxide-Nanoparticles-Atef Oraby/3a22cb5c68f2ac0f2a732eda03d657 ca01bfe8be

16. Velayutham K, Rahuman AA, Rajakumar G, Santhoshkumar T, Marimathu S, Javaseelan $\mathrm{C}$, et al. "Evaluation of Catharanthus roseus leaf extract-mediated biosynthesis of titanium dioxide nanoparticles against Hippobosca maculata and Bovicola ovis. Parasitol Res 2012; 111(6):2329-2337. https://doi. org/10.1007/s00436-011-2676-x
17. Noori A, Karimi F, Fatahian S, Yazdani $F$. Effects of zinc oxide nanoparticles on renal function in mice. Int J Biosci 2014; 5(9):140-146. http://doi.org/10.12692/ $\mathrm{ijb} / 5.9 .140-146$

18. Mody VV, Siwale R, Singh A, Mody HR. Introduction to metallic nanoparticles. J Pharm Bioallied Sci 2010; 2(4):282-289. https://doi.org/10.4103/0975-7406.72127

19. Sondi I, Salopek-Sondi B. Silver nanoparticles as antimicrobial agent: A case study on $E$. coli as a model for Gram-negative bacteria. J Colloid Interface Sci 2004; 275(1):177-182. https://doi.org/10.1016/j.jcis.2004.02.012

20. Banumathi B, Malaikozhundan B, Vaseeharan B. In vitro acaricidal activity of ethnoveterinary plants and green synthesis of zinc oxide nanoparticles against Rhipicephalus (Boophilus) microplus. Vet Parasitol 2016; 30(216):93-100. https:// doi.org/10.1016/j.vetpar.2015.12.003

21. Bogdanchikova N, Vázquez-Muñoz R, HuertaSaquero A. Silver nanoparticles composition for treatment of distemper in dogs. Int J Nanotecnology 2016; 13(1-3):225-235. https://tpu.pure.elsevier.com/en/publications/ silver-nanoparticles-composition-fortreatment-of-distemper-in-do

22. Wójcik M, Lewandowwski W, Król M, Pawlowski $\mathrm{K}$, Mieczkowski J, Lechowski R, et al. Enhancing anti-tumor efficacy of doxorubicin by noncovalent conjugation to gold nanoparticles-In vitro studies on feline fibrosarcoma cell lines. PLoSOne 2015; 10(4):e0129639. https://doi. org/10.1371/journal.pone.0124955

23. Wong KKY, Cheung SO, Huang L, Niu J, Tao $\mathrm{C}$, Ho CM, et al. Further evidence of the antiinflammatory effects of silver nanoparticles. Chem Med Chem 2009; 4(7):1129-1135. https://doi.org/10.1002/cmdc.200900049

24. Yaqoob AA, Ahmad H, Parveen T, Ahmad A, Oves M, Ismail IMI, etal. Recent advances in metal decorated nanomaterials and their various biological applications: A review. Front Chem 2020; 8(341):1-23. https:// doi.org/10.3389/fchem.2020.00341 
25. Kuswandi B, Futra D, Heng LY. Chapter 15-Nanosensors for the detection of contaminants. In: Nanotechnology Application in Food; FLavor Stability, Nutrion and Safety 2017. https://doi.org/10.1016/ B978-0-12-811942-6.00015-7

26. Tomar RS, Preet S. Evaluation of anthelmintic activity of biologically synthesized silver nanoparticles against the gastrointestinal nematode, Haemonchus contortus. J Helminthol. 2016;91(4):454-461. https:// doi.org/10.1017/S0022149X16000444

27. Saleh M, Kumar G, Abdel-Baki AA, Al-quraishy $\mathrm{S}$, El-matbouli M. In vitro antimicrosporidial activity of gold nanoparticles against Heterosporis saurida. BMC Vet Res 2016;12(44):1-6. https://doi.org/10.1186/ s12917-016-0668-X

28. Pimentel-Acosta CA, Morales-Serna FN, Chávez-Sánchez Mc, Lara $\mathrm{HH}$, Pestryakov A, Bogfanchikova N, et al. Efficacy of silver nanoparticles against the adults and eggs of monogenean parasites of fish. Parasitol Res 2019; 118(6):1741-1749. https://doi. org/10.1007/s00436-019-06315-9

29. Saleh M, Abdel-Baki AA, Dkhil MA. ElMatbouli M, Al-Quraishy S. Antiprotozoal effects of metal nanoparticles against Ichthyophthirius ultifiliis. Parasitology 2017; 44(13):1802-1810. https://doi. org/10.1017/S0031182017001184

30. Miraballes C, Riet-Correa F. A review of the history of research and control of Rhicephalus (Boophilus) microplus, babebiosis and anaplasmosis in Uruguay. Exp Appl Acarol. 2018; 75(4):383-398 https:// doi.org/10.1007/s10493-018-0278-3

31. Benelli G. Mode of action nanoparticles against insects. Environ Sci Pollut Res. 2018; 25(13):12329-12341. https://doi. org/10.1007/s11356-018-1850-4

32. Marimuthu S, Rahuman AA, Rajakumar G, Santhoshkumar T, Kirthi AV, Jayaseelan C, et al. Evaluation of green synthesized silver nanoparticles against parasites. Parasitol Res. 2011; 108(6):1541-1549. https://doi. org/10.1007/s00436-010-2212-4
33. Banumathi $B$, Vaseeharan $B$, Malaikozhundan B, Ramasamy P, Govindarajan M. Alharbi NS, et al., Green larvacides aganist blowflies, Lucilia sericata (Diptera Calliphoridae): Screening of seven plants used in Indian ethno-veterinary medicine and production of green-coated zinc oxide nanoparticles. Physiol Mol Plant Pathol. 2018; 101:214218. https://doi.org/10.1016/j. pmpp.2017.02.003

34. Marimuthu S, Rahuman AA, Santhoshkumar T, Jayaseelan C, Kirhi AV Bagavan A, et al. Lousicidal activity of synthesized silver nanoparticles using Lawsonia inermis leaf aqueous extract against Pediculus humanus capitis and Bovicola ovis. Parasitol Res. 2012; 111(5):2023-2033. https://doi. org/10.1007/s00436-011-2667-y

35. El-Diasty EM, Ahmed MA, Okasha N, Mansour S, El-Dek SI, El-Khalek HMABD Youssif $\mathrm{MH}$. Antifungal activity of zinc oxide nanoparticles against dermatophytic lesions of cattle. Rom J Biophys. 2013; 23(3):191202. https://doi.org/10.5897/AJB11.1499

36. Yuan Y, Peng Q, Gurunathan S. Effects of Silver nanoparticles on multiple drugresistant strains of Staphylococcus aureus and Pseudomonas aeruginosa from mastitisinfected goats: An alternative approach for antimicrobial therapy. Int J Mol Sci. 2017; 18(3):2-22. https://doi.org/10.3390/ ijms18030569

37. Gurunathan S, Choi. YJ, Kim JH. Antibacterial efficacy of silver nanoparticles endometritis caused by Prevotella melaninogenica and Arcanobacterum pyogenes in dairy cattle. Int J Mol Sci 2018; 19(4):1210. https://doi. org/10.3390/ijms19041210

38. Mohamed MM, Fouad SA Elshoky HA, Mohammed GM, Salaheldin TA. Antibacterial effect of gold nanoparticles against Corynebacterium pseudotuberculosis. Int J Vet Sci Med. 2017; 5(1):23-29. https:// doi.org/10.1016/j.ijvsm.2017.02.003

39. Ortiz-Arana G. Evaluación del efecto bactericida in vitro de las nanopartículas de plata en cepas de Moraxella spp multirresistentes aisladas en ovinos en el Estado de México [Tesis de maestría]. Toluca, México: Universidad Autónoma del Estado de México; 2019. http:// hdl.handle.net/20.500.11799/105338 
40. Bansod SD, Bawaskar MS, Gade AK., Rai MK. Development of shampoo, soap and ointment formulated by green synthesised silver nanoparticles functionalised with antimicrobial plants oils in veterinary dermatology: Treatment and prevention strategies. IET Nanobiotechnology 2015; 9(4):165-171. http://doi.org/10.1049/ietnbt.2014.0042

41. Flores-González M, Talavera-Rojas M, Soriano-Vargas $\mathrm{E}$, Rodríguez-González V. Practical mediated-assembly synthesis of silver nanowires using commercial: Camellia sinensis extracts and their antibacterial properties. New J Chem 2019;42(3):21332139. https://doi.org/10.1039/C7NJ03812G

42. Soltani M, Ghodratnema M, Ahari H, Mousavi $\mathrm{EH}$, Atee M, Dastmalchi F, Rahmanya $J$, et al. The inhibitory effect of silver nanoparticles on the bacterial fish pathogens, Streptococcus iniae, Lactococcus garvieae, Yersinia ruckeri and Aeromonas hydrophila. Int J Vet Res. 2009; 3(2):137-142. https://pdfs.semanticscholar.org/8c29/ f52e713f347c4630f59b134b62fdc0f56d0.pdf

43. Shaalan MI, El-Mahdy MM, Theiner S, ElMatbouli M, Saleh M. In vitro assessment of the antimicrobial activity of silver and zinc oxide nanoparticles against fish pathogens. Acta Vet Scand. 2017; 59(1):1-11. https:// doi.org/10.1186/s13028-017-0317-9

44. Rafiei S, Rezatofighi SE, Ardakani MR, Madadgar $O$. In vitro anti-foot-and-mouth disease virus activity of magnesium oxide nanoparticles. IET Nanobiotechnol. 2015; 9(5):247-251. http://dx.doi.org/10.1049/ iet-nbt. 2014.0028

45. Wójcik M, Lewandowski W, Król M, Pawlowski K, Mieczkowski J, Lechowski R, et al. Correction: Enhancing anti-tumor efficacy of doxorubicin by non-covalent conjugation to gold nanoparticles- In vitro studies on feline fibrosarcoma cell lines. PLoS One $2015 ; 10(6)$ :e0129639. https://doi. org/10.1371/journal.pone.0129639
46. Zabielska-Koczywąs K, Wojtalewicz A,Uzarowska E, Klejman A, Wojtkowska A, et al. Distribution of glutathione-stabilized gold nanoparticles in feline fibrosarcomas and their role as a drug delivery system for doxorubicin-preclinical studies in a murine model. Int J Mol Sci. 2018; 19(4):1-19. https://doi.org/10.3390/ijms19041021

47. Sincai M, Ganga D, Ganga M, Argherie D, Bica $D$. Antitumor effect of magnetite nanoparticles in cat mammary adenocarcinoma. J Magn Magn Mater. 2005; 293(1):438-441. https:// doi.org/10.1016/j.jmmm.2005.02.074

48. Gurunathan S, Han JW, Park JH, Kim E, Choi YJ, Kwon DN, et al. Reduced graphene oxide-silver nanoparticle nanocomposite: a potential anticáncer nanotherapy. Int J Nanomedicine 2015; 10(6):6257-6276. https://doi.org/10.2147/IJN.S92449

49. Scott NR. Nanotechnology and animal health. Rev Sci Tech. 2005; 24(1):425-432. http://doi.org/10.20506/rst.24.1.1579

50. Kuswandi B, Futra D, Heng LY. Chapter 15-Nanosensors for the detection of contaminants. In: Nanotechnology Application in Food; FLavor Stability, Nutrion and Safety. 2017:307-333. https://doi. org/10.1016/B978-0-12-811942-6.00015-7

51. Pineda L, Chwalibog A, Sawosz E, Lauridsen C, Engberg R, Elnif J, et al. Effect of silver nanoparticles on growth performance, metabolism and microbial profile of broiler chickens. Arch Anim Nutr. 2012; 66(5):416429. https://doi.org/10.1080/174503 9X.2012.710081

52. Bhanja SK, Hotowt A, Mehra M, Sawosz $E$, Pineda $L$, Vadalasetty $K P$, et al. In ovo administracion of silver nanoparticles and/ or amino acids influence metabolism and immune gene expresion in chicken embryos. Int J Mol Sci. 2015; 16(5):9484-9503. https://doi.org/10.3390/ijms16059484

53. Romero-Pérez A, García-García E, Zavaleta-Mancera A, Ramírez-Bribiesca J, Revilla-Vázquez A, Hernández-Calva, et al. Designing and evaluation of sodium selenite nanoparticles in vitro to improve selenium absorption in ruminants. Vet Res Commun. 2010; 34(1):71-79. https://doi. org/10.1007/s11259-009-9335-z 
54. Tayel AA, El-Tras WF, Moussa S, El-Baz AF, Mahrous $H$, Salem MF, et al., Antibacterial action of zinc oxide nanoparticles against foodborne pathogens. J Food Saf. 2010; 31(2):211-218. https://doi.org/10.1111/ j.1745-4565.2010.00287.x

55. Ashraf AAET, Ahmed MA, Diasty EM, Fatma IEH, Ahmed Youssef MM. A comparative study on antifungal activity of $\mathrm{FE}_{2} \mathrm{O}_{3}$, and $\mathrm{FE}_{3} \mathrm{O}_{4}$ nanoparticles. Int J Adv Res. 2018; 6(1):189194. http://doi.org/10.21474/IJAR01/6204

56. Hassan AR, de la Escosura- Muñiz A, Merkoçi A, Highly sensitive and rapid determination of Escherichia coli 0157:H7 in minced beef and wáter using electrocatalytic gold nanoparticle tags. Biosens Biolectron 2015; 67:511-515. https://doi.org/10.1016/j. bios.2014.09.019

57. Giovannozzi AM, Rolle F, Sega M, Abete MC, Marchis D, Rossi AM. Rapid and sensitive detection of melamine in milk with gold nanoparticles by Surface Enhanced Raman Scattering. Food Chem. 2014; 159:250-256. https://doi.org/10.1016/j. foodchem.2014.03.013

58. Kim HJ, Kim SH, Lee JK, Choi CU, Lee HS, Kang HG, et al. A novel mycotoxin purification system using magnetic nanoparticles for the recovery of aflatoxin B1 and zearalenone from feed. J Vet Sci. 2012; 13(4):363-369. http://doi.org/10.4142/jvs.2012.13.4.363

59. Cao Y, Ma Y, Zhang M, Wang H, Tu X, Shen H, et al. Ultrasmall graphene oxide supported gold nanoparticles as adjuvants improve humoral and cellular immunity in mice. Adv Funct Mater. 2014; 24(44):6963-6971. https://doi.org/10.1002/adfm.201401358

60. Asgary V, Shoari A, Baghbani-Arani F, Sadat Shandiz SA, Khosravy MS, Janani $A$, et al. Green synthesis and evaluation of silver nanoparticles as adjuvant in rabies veterinary vaccine. Int J Nanomedicine 2016; 11:3597-3605. https://doi.org/10.2147/ IJN.S109098
61. Staroverov SA, Volkov AA, Larionov SV, Mezhennyy PV, Kozlov S, Fomin AS, et al. Study of transmissible-gastroenteritisvirus-antigen-conjugated immnunogenic properties of selenium nanoparticles and gold. Life Sci J 2014; 11(11):456-460. http://www.lifesciencesite.com/lsj/life111 1/078 25876life111114 456 460.pdf

62. Ning $P$, Wu $Z$, Li $X$, Zhou $Y$, Hu A, Gong $X$, et al. Development of functionalized gold nanoparticles as nanoflare probes for rapid detection of classical swine fever virus. Colloids Surfaces B Biointerfaces. 2018; 1(171):110-114. https://doi.org/10.1016/j. colsurfb.2018.07.024

63. Stringer RC, Schommer S, Hoehn D, Grant SA. Development of an optical biosensor using gold nanoparticles and quantum dots for the detection of Porcine Reproductive and Respiratory Syndrome Virus. Sens Actuator B-Chem. 2008; 134(2):427-431. https://doi.org/10.1016/j.snb.2008.05.018

64. Sattarahmady N, Tondro GH., Gholchin M, Heli H. Gold nanoparticles biosensor of Brucella spp. genomic DNA: Visual and spectrophotometric detections. Biochem Eng J. 2015;97(15):1-7. https://doi. org/10.1016/j.bej.2015.01.010

65. Saleh M, Soliman H, Haenen O, El-Matbouli M. Antibody-coated gold nanoparticles immunoassay for direct detection of Aeromonas salmonicida in fish tissues. J Fish Dis. $2011 ; 34(11): 845-852$. https://doi. org/10.1111/j.1365-2761.2011.01302.x 